\title{
The immune status in situ of recurrent tonsillitis and idiopathic tonsillar hypertrophy
}

\author{
Semberova $\mathrm{J}^{1}$, Rychly $\mathrm{B}^{2}$, Hanzelova $\mathrm{J}^{3}$, Jakubikova $\mathrm{J}^{4}$ \\ Department of Clinical Immunology and Allergology, Children's University Hospital, Bratislava, Slovakia. \\ jana semberova@dfnsp.sk
}

\begin{abstract}
Objective: To analyze the immune status in situ of tonsils of patients with recurrent tonsillitis (RT) and idiopathic tonsillar hypertrophy (ITH) with the aim to discuss the indications of tonsillectomy (TE) and tonsillotomy (TT) in young children.

Methods: The histological and immunohistological study of tonsillar tissue of RT and ITH in correlation with immunological parameters in peripheral blood in 13 patients with RT and 16 patients with ITH.

Results: In the RT group, we found a higher degree of fibrosis with a higher density of memory lymphocytes $\left(\mathrm{CD} 45 \mathrm{RO}^{+}\right)$, B-lymphocytes $\left(\mathrm{CD} 20^{+}\right)$and cytotoxic T-lymphocytes $\left(\mathrm{CD}^{+}\right)$in surface epithelium of tonsils compared to the ITH group (NS). The density of immunoglobulin IgG in the crypt epithelium in RT was significantly higher than in the ITH group $(p=0.041)$. We also measured a higher sera concentration of immunoglobulines ( $\lg G, \lg M, \lg A$ ) and TNF- $\alpha$ in RT compared to the ITH group (NS) and $T_{H^{-1}}$ immune response in tonsillar tissue based on differences between local cytokine concentration TNF- $\alpha$ and IL-4.

Conclusions: RT has a higher inflammatory reaction in tonsillar tissue as a result of persistent bacterial antigenic stimulation. In patients with RT, the tonsillectomy might be the only option for surgical treatment. In patients with ITH with mild symptoms, the tonsillotomy should be preferred (Tab. 3, Ref. 24). Full Text in PDF www.elis.sk. Key words: recurrent tonsillitis, idiopathic tonsillar hypertrophy, immunohistochemistry, histology, immunity.
\end{abstract}

Epidemiological trends in the numbers of TE have increased rapidly and the surgical indications have shifted from infection to upper airway obstruction $(1,2)$. In younger children, the obstruction of airways without infection is the main indication for this procedure, with the age the infection becomes more prominent indication (3)

Tonsillotomy (TT) is accepted as a safe and effective method for treatment of obstructive sleep-related respiratory disturbances in children (4). In tonsillar hyperplasia even without history of infection the chronic inflammation can be found histologically (5) and there is a low risk of regrowth of tonsillar tissue after TT $(4,5)$.

That's the reason why the indication for these ENT procedures are being discussed among ENT clinicians and pediatricians. In the present study we have investigated the immune status in situ in patients with RT and ITH.

\section{Patients and methods}

\section{Patients}

For this study we have selected 13 patients with RT (10 boys, 3 girls in the age $2-12$ years, mean 5.87 years \pm 2.346$)$ and 16

${ }^{1}$ Department of Clinical Immunology and Allergology, Children's University Hospital, Bratislava, Slovakia, ${ }^{2}$ Cytopathos, s. r. o., Bratislava, Slovakia, ${ }^{3} 1$ st Otolaryngology Clinic, University Hospital, Bratislava, Slovakia, and ${ }^{4}$ Pediatric Otorhinolaryngology Department, Children's University Hospital, Bratislava, Slovakia

Address for correspondence: J. Semberova, Dept of Clinical Immunology and Allergology, Children's University Hospital, Limbova 1, SK-833 40 Bratislava, Slovakia.

Phone: +421.2 .259371538$ patients with ITH (13 boys, 3 girls in the age $3-8$ years, mean $4.67 \pm 1.779$ years) who underwent surgery of tonsils under general anaesthesia at the ENT clinics in the time period November 2007 - June 2008.

All thirteen patients with RT had history of tonsillitis with more than 4 episodes per year - they underwent tonsillectomy. The patients with ITH had history of obstructive symptoms or history of sleep apnea syndrome with no history of recurrent infections. Ten patients with ITH (62.5\%) underwent TT and six of them (37.5\%) underwent TE.

The patients with the history of known allergies, the history of antibiotic or antihistaminic drugs taken at least 1 month before surgery, or patients with suspicion of malignant disease were not included in the study. All patients had no evidence of clinical infection at the time of surgery.

Before the surgery, the blood needed for immunological investigation was taken.

All procedures were performed in accordance with the ethical guidelines of the Children's University Hospital in Bratislava. A signed written consent was obtained from parents of the patients.

\section{Histological investigation}

Each tonsil specimen was divided into 2 parts - one section was preserved in $10 \%$ buffered formaline at room temperature for 24 hours routinely processed in automatic tissue processor and then embeded in paraffin blocks. Five ųm thick slices were stained with hematoxylin and eosin for histological assessment. The second section was prepared for immunohistochemical investigation. Additionally, in 2 patients with RT 1 gram of tonsillar tissue was 
homogenized in $2 \mathrm{ml}$ of physiological solution and than centrifuged at $3000 \mathrm{rpm}$ for 10 minutes. The supernatant was kept at $-20{ }^{\circ} \mathrm{C}$ until the cytokines were analyzed (Flowcytomix, Bender Medsystems, Austria).

\section{Immunohistochemistry}

Sections were processed using antihuman monoclonal antibodies according to the peroxidase-antiperoxidase method. Following cell surface antigens were used:

(i) examination of memory lymphocytes $\mathrm{CD} 45^{+} \mathrm{R} 0^{+}$(DAKO, $1: 150)$

(ii) examination of cytotoxic T-lymfocytes $\mathrm{CD} 8^{+}$(DAKO, RTU)

(iii) examination of B-lymfocytes CD20 $0^{+}$(DAKO, 1:400)

(iv) examination of immunoglobulin $\operatorname{IgA}$ and $\operatorname{IgG}$ (LAB VISION, RTU)

\section{Immunological investigation}

The blood samples were taken in both groups of patients before ENT surgery.

The subpopulations of lymphocytes were determined by flow cytometry using monoclonal antibodies (BeckmanCoulter FC 500) for total T lymphocytes $\left(\mathrm{CD}^{+}\right)$, helper T-lymphocytes $\left(\mathrm{CD}^{+}\right)$, cytotoxic T-lymphocytes $\left(\mathrm{CD} 8^{+}\right)$, B-lymphocytes $\left(\mathrm{CD} 19^{+}\right)$. Immunoglobulines were analyzed in serum by nephelometry.

In the RT group, the cellular immunity was evaluated in 10 patients $(76.9 \%)$, humoral immunity in 11 patients $(84.6 \%)$. In ITH group, the subpopulations of lymphocytes were stained in 14 patients $(87.5 \%)$, the immunoglobulines in 11 patients $(68.7 \%)$.

The TNF- $\alpha$ in serum was measured in 4 patients with RT and in 3 patients with ITH by ELISA (Human TNF- $\alpha$ instant ELISA, Bender Medsystems, Austria).

\section{Histological and immunohistochemical evaluation}

We evaluated histological specimens in 15 patients with ITH $(93.7 \%)$ and 12 patients with RT $(92.3 \%)$.

Each section was assessed in the light microscope with original magnification 400x. We evaluated the surface epithelium according to the pathology classification $(0-$ absence or 1 - presence of keratosis / parakeratosis, oedema, infiltration of bacteria, polymorphonuclear leukocytes (PMN), the degree of fibrosis 1 - light, 2 severe), the crypts and the number of follicles per high power field.

We performed a semiquantitative analysis using the following scale for the density of cells positive for $\mathrm{CD} 45 \mathrm{R} 0^{+}, \mathrm{CD} 20^{+}, \mathrm{CD}^{+} \mathrm{s}$ and immunoglobulines IgG, IgA: 0 - absence, 1 - mild density ( 0 $33 \%$ ), 2 -moderate density (33-66\%), 3- severe density (> $66 \%$ ).

\section{Statistical analysis}

The chi quadrat test and Fischer exact test were performed to compare the presence or absence of histological and immunohistochemical findings in cross tables.

For 2-group comparison analyse, the Student t-test (for normal distribution) or Mann-Whitney test were performed (if the material was not normally distributed). $\mathrm{p}$ values less than 0.05 were considered to be significant. We used the SPSS for Windows statistical packet program.

\section{Results}

\section{Histological investigation}

The patients with RT had 1.66 times more often a higher degree of fibrosis (degree 2) in the surface epithelium compared to the ITH group (33.3\% vs $20 \%$ ). The patients with ITH had 1.30 times more often oedema in the surface epithelium than the RT group $(86.7 \%$ vs $66.7 \%)$.

There was no difference in the presence of parakeratosis or keratosis in the epithelium ( $66.7 \%$ in both groups of patients), intraepithelial colonization of bacteria $(26.7 \%$ in the ITH group, $25 \%$ in the RT group) and intraepithelial infiltration of polymorphonuclear leukocytes (60\% in the ITH group, $66.7 \%$ in the RT group). Almost in all patients of both groups, there was fibrin, lymphocytes and polymorphonuclear leukocytes observed in the crypts. The number of follicles per high power field was found to be similar in both groups - in the ITH group the mean was 12.87 \pm 4.955 , in the RT group $13.58 \pm 4.144$. The results were not statistically significant (Tab. 1).

\section{Immunohistological investigation}

In the RT group, there was 1.58 times more often higher density of memory lymphocytes (density $2+3$ ) in the surface epithelium of tonsils (69.3\% vs $43.8 \%$ ) and 1.37 times higher in the crypt epithelium (76.9\% vs $56.3 \%$ ) compared to the ITH group. In the surface epithelium, there was also 6-times more often higher density of cytotoxic T-lymphocytes (density $2+3,38.5 \%$ vs 6.3 $\%$ ) and 1.54 times more B-lymphocytes (density $2+3,38.5 \%$ vs $25.0 \%$ ) in RT comparing ITH group.

In both groups of patients there was a higher density of Blymphocytes in the crypts than in surface epithelium - in the RT group 2.4 times higher $(92.3 \% / 38.5 \%)$ and in the ITH group 3.5 times higher $(87.5 \%$ / 25\%). The results were not significant.

The patients with RT had a statistically significant higher density of staining of immunoglobulin IgG under the crypts $(p=0.041)$.

Tab. 1. Histological investigation of tonsils.

\begin{tabular}{|c|c|c|c|c|c|c|c|c|c|c|c|c|}
\hline \multirow{2}{*}{\multicolumn{2}{|c|}{ Group }} & \multicolumn{2}{|c|}{ Keratosis } & \multicolumn{2}{|c|}{ Oedema } & \multicolumn{3}{|c|}{ Fibrosis } & \multicolumn{2}{|c|}{ Bacteria } & \multicolumn{2}{|c|}{ PMN } \\
\hline & & 0 & 1 & 0 & 1 & 0 & 1 & 2 & 0 & 1 & 0 & 1 \\
\hline \multirow[t]{2}{*}{$\overline{\mathrm{RT}}$} & N. & 4 & 8 & 4 & 8 & 1 & 7 & 4 & 9 & 3 & 4 & 8 \\
\hline & $\%$ & $33.3 \%$ & $66.7 \%$ & $33.3 \%$ & $66.7 \%$ & $8.3 \%$ & $58.3 \%$ & $33.3 \%$ & $75.0 \%$ & $25 \%$ & $33.3 \%$ & $66.7 \%$ \\
\hline \multirow[t]{2}{*}{$\overline{\mathrm{ITH}}$} & N. & 5 & 10 & 2 & 13 & 3 & 9 & 3 & 11 & 4 & 6 & 9 \\
\hline & $\%$ & $33.3 \%$ & $66.7 \%$ & $13.3 \%$ & $86.7 \%$ & $20.0 \%$ & $60.0 \%$ & $20.0 \%$ & $73.3 \%$ & $26.7 \%$ & $40.0 \%$ & $60.0 \%$ \\
\hline \multicolumn{2}{|c|}{$\mathrm{P}$ value } & \multicolumn{2}{|c|}{1.000} & \multicolumn{2}{|c|}{0.213} & \multicolumn{3}{|c|}{0.568} & \multicolumn{2}{|c|}{1.000} & \multicolumn{2}{|c|}{1.000} \\
\hline
\end{tabular}

Abbreviations: $\mathrm{N}$ - number of patients, 0 - absense, 1- light degree, 2 - severe degree of histological changes 
Tab. 2. Immunohistochemical investigation of tonsils.

\begin{tabular}{|c|c|c|c|c|c|c|c|c|c|c|c|c|}
\hline \multirow{5}{*}{$\begin{array}{l}\text { Localisation } \\
\text { density } \\
\text { ITH N. } \\
\%\end{array}$} & \multicolumn{12}{|c|}{ CD45R0+ lymphocytes } \\
\hline & \multicolumn{3}{|c|}{ Surface epithelium } & \multicolumn{3}{|c|}{ In crypts } & \multicolumn{3}{|c|}{ Extrafollicular area } & \multicolumn{3}{|c|}{ Intrafollicular } \\
\hline & 1 & 2 & 3 & 1 & 2 & 3 & 1 & 2 & 3 & 1 & 2 & 3 \\
\hline & 9 & 3 & 4 & 7 & 8 & 1 & 2 & 11 & 3 & 7 & 8 & 1 \\
\hline & 56.3 & 18.8 & 25.0 & 43.8 & 50.0 & 6.3 & 12.5 & 68.8 & 18.8 & 43.8 & 50.0 & 6.3 \\
\hline RT N. & 4 & 6 & 3 & 3 & 7 & 3 & 1 & 9 & 3 & 4 & 8 & 1 \\
\hline$\%$ & 30.8 & 46.2 & 23.1 & 23.1 & 53.8 & 23.1 & 7.7 & 69.2 & 23.1 & 30.8 & 61.5 & 7.7 \\
\hline \multirow[t]{2}{*}{$\mathrm{p}$ value } & & 0.260 & & & 0.346 & & & 1.000 & & & 0.844 & \\
\hline & \multicolumn{6}{|c|}{ CD8 ${ }^{+}$lymphocytes } & \multicolumn{6}{|c|}{ CD20+1ymphocytes } \\
\hline Localisation & \multicolumn{3}{|c|}{ Surface epithelium } & \multicolumn{3}{|c|}{ Extrafollicular area } & \multicolumn{3}{|c|}{ Surface epithelium } & \multicolumn{3}{|c|}{ In crypts } \\
\hline$\overline{\text { density }}$ & 1 & 2 & 3 & 1 & 2 & 3 & 1 & 2 & 3 & 1 & 2 & 3 \\
\hline ITH N. & 15 & 1 & 0 & 5 & 6 & 5 & 12 & 4 & 0 & 2 & 10 & 4 \\
\hline$\%$ & 93.8 & 6.3 & 0.0 & 31.3 & 37.5 & 31.3 & 75.0 & 25.0 & 0.0 & 12.5 & 62.5 & 25.0 \\
\hline RT N. & 8 & 3 & 2 & 4 & 4 & 5 & 8 & 4 & 1 & 1 & 7 & 5 \\
\hline$\%$ & 61.5 & 23.1 & 15.4 & 30.8 & 30.8 & 38.5 & 61.5 & 30.8 & 7.7 & 7.7 & 53.8 & 38.5 \\
\hline \multirow[t]{2}{*}{ p value } & & 0.096 & & & 1.000 & & & 0.535 & & & 0.865 & \\
\hline & \multicolumn{12}{|c|}{ immunoglobulin IgG } \\
\hline Localisation & \multicolumn{3}{|c|}{ In crypts } & \multicolumn{3}{|c|}{ Surface epithelium } & \multicolumn{3}{|c|}{ Intrafollicullar } & \multicolumn{3}{|c|}{ Extrafollicular } \\
\hline$\overline{\text { density }}$ & 1 & 2 & 3 & 1 & 2 & 3 & 1 & 2 & 3 & 1 & 2 & 3 \\
\hline ITH N. & 8 & 5 & 3 & 3 & 6 & 7 & 12 & 3 & 1 & 10 & 5 & 1 \\
\hline$\%$ & 50.0 & 31.3 & 18.8 & 18.8 & 37.5 & 43.8 & 75.0 & 18.8 & 6.2 & 62.5 & 31.3 & 6.3 \\
\hline RT N. & 1 & 9 & 3 & 4 & 3 & 6 & 8 & 2 & 3 & 7 & 6 & 0 \\
\hline$\%$ & 7.7 & 69.2 & 23.1 & 30.8 & 23.1 & 46.2 & 61.5 & 15.4 & 23.1 & 53.8 & 46.2 & 0.0 \\
\hline p value & & 0,041 & & & 0.721 & & & 0.451 & & & 0.835 & \\
\hline
\end{tabular}

Abbreviations: RT - recurrent tonsillitis, ITH - idiopathic tonsillar hypertrophy, $\mathrm{N}$ - number of patients, $\%$ - percentage of positive cells, 1 - mild density, 2 - moderate density, 3 - severe density.

Tab. 3. Cytokines in tissue supernatant in RT.

\begin{tabular}{lcccccc}
\hline Cytokine pg/ml & IL-10 & IL-8 & IL-4 & IL-1 $\beta$ & TNF- $\alpha$ & TNF- $\beta$ \\
\hline patient 1 & 92.6 & 4.180 & 17.07 & 3.549 & 467.87 & 15.89 \\
patient 2 & 172.89 & 4.071 & 0 & 4.561 & 430.47 & 41.25 \\
\hline
\end{tabular}

No patient had grade zero of immunohistochemical markers (Tab. 2).

\section{Immunological investigation}

The patients with RT had a higher sera concentrations of immunoglobulines $\operatorname{IgG}$ (in the RT group the mean was $10.16 \pm 3.655$ $\mathrm{g} / \mathrm{l}$, in the ITH group the mean was $7.49 \pm 2.240 \mathrm{~g} / \mathrm{l}$ ), $\operatorname{IgA}$ (in the RT group the mean was $1.03 \pm 0.760 \mathrm{~g} / \mathrm{l}$, in the ITH group the mean was $0.96 \pm 0.657 \mathrm{~g} / \mathrm{l}$ ), $\operatorname{IgM}$ (in the RT group the mean was $1.95 \pm 2.698 \mathrm{~g} / \mathrm{l}$, in the ITH group the mean $0.79 \pm 0.335 \mathrm{~g} / \mathrm{l}$ ).

We found no significant differences in subpopulations of lymphocytes in peripheral blood between both patient groups: $\mathrm{CD}^{+}$lymphocytes (RT mean $69.18 \% \pm 4.925$, ITH mean 63.7 $\% \pm 9.857$ ), $\mathrm{CD}^{+}$lymphocytes (RT mean $33.96 \% \pm 3.897, \mathrm{ITH}$ mean $31.14 \pm 7.775$ ), $\mathrm{CD}^{+}$lymphocytes (RT mean $25.06 \% \pm$ 4.389, ITH mean $26.36 \% \pm 5.678)$ and CD19+ lymphocytes (RT mean $19.29 \% \pm 5.882$, ITH mean $20.86 \% \pm 7.539$ ).

The higher sera concentrations of TNF- $\alpha$ in patients with RT than in patients with ITH were also measured (mean $10.03 \pm 3.489$ $\mathrm{pg} / \mathrm{ml}$ in the RT group, $6.89 \pm 3.856 \mathrm{pg} / \mathrm{ml}$ in the ITH group).

In two patients with RT, the cytokines in tissue supernatant were analyzed. The cytokines IL-2, IL-5, IL-6, IL-12p70 were not detected in the samples. The highest concentration was measured for cytokine TNF- $\alpha$ (mean $449.17 \mathrm{pg} / \mathrm{ml})$.
The results of immunological investigation of peripheral blood and tissue supernatant were not statistically significant (Tab. 3).

\section{Discussion}

The palatine tonsils as the part of Waldeyer's ring are located at the gateway of gastrointestinal and respiratory tract where they are continually being bombarded with antigens entering the respiratory and gastrointestinal tract. The epithelial area exposed to antigen is maximized by $10-30$ blind-ending crypts extending deeply into tonsillar tissue. The tonsils consist of four lymphoid compartments: the reticular crypt epithelium, the extrafollicular area, the mantle zones of lymphoid follicles and the germinal centres. Within the antigen is taken up by antigen-presenting cells of the macrophage lineage in crypt epithelium, it is presented to Tcells and consequently the cell-mediated and humoral responses are inducted (6).

The aetiology of ITH is not well understood. The increase of B- and T-lymphocytes was reported in recurrent tonsillitis with tonsillar hypertrophy (RTTH) compared to RT (8). There might be a deficiency of proliferating active cells in response to mitogenic stimulation in RTTH (9).

In the present study we have investigated the immune status in situ in patients with RT and ITH in correlation with histological and immunological investigation of peripheral blood.

In comparison of both patients groups we found a higher degree of epithelial fibrosis in the RT group. Other authors also reported fibrosis in the RT group $(5,7)$. The fibrosis of the tonsillar epithelium in RT lead to impaired barrier function of the tonsils with a local dysfunction of immunity what consequently caused the re- 
current infections (7). These histological changes of the tonsillar epithelium as parakeratosis or keratosis can be also observed in both patient groups. Alatas reported no difference in the epithelial fibrosis between patients with RT and RTTH (9). These different findings might be due to different groups of patients, because the patients with ITH in our study didn't present with the history of recurrent infection and the higher degree of epithelial fibrosis in our RT group was a result of recurrent infections. The epithelial fibrosis causes an impaired antigen uptake and this in turn leads to the recurrence of inflammatory process.

In the ITH group, the epithelial oedema of the surface epithelium might be due to non-specific bacteria-induced release of histamin (10) or due to local allergic reaction to bacterial or food allergens (11).

Previous studies observed that ITH occurs with follicular hyperplasia $(9,12)$ and RT with a higher number of follicules $(9,13)$. We didn't measure the follicular area by histological investigation and we didn't observe any significant changes in follicular number between patients with RT and ITH.

The immunohistochemical studies differ in analyzing subpopulations of lymphocytes in patients with RT and ITH. There are not many studies evaluating cellular immunity in different lymphoid compartments in tonsillar tissue.

Rosenmann et al detected no significant changes in subpopulations of T- and B-cells in tonsils and peripheral blood between patients with ITH and RT (14). Brodsky reported that the infection and bacterial load led to increase of T- and B-lymphocytes in RTTH compared to RT (8).

We investigated in patients with RT a higher density (degree $2+3)$ of memory lymphocytes $\left(\mathrm{CD} 45 \mathrm{R} 0^{+}\right)$in surface epithelium and in the crypt epithelium compared to the ITH group. In surface epithelium, there was also a higher density of cytotoxic T-lymphocytes $\left(\mathrm{CD}^{+}\right)$and B-lymphocytes $\left(\mathrm{CD} 20^{+}\right)$in RT comparing to the ITH group. The patients with RT had a statistically significantly higher density of staining of immunoglobulin IgG under the crypts. There was a higher density of B-lymphocytes in crypts than in surface epithelium in both groups. The observed findings showed that crypt epithelium in the case of RT had more active role in specific immune response than in the ITH group. Crypt epithelium may have an epithelial barrier different from surface epithelium, the integrity of cryptal epithelium is important for immune function of palatine tonsils as a defense organ against infections (15).

Our results are only partially comparable with those of Alatas (9), but there are clear differences in the target patient groups. He observed in the RTTH group a higher density of B-lymphocytes $\left(\mathrm{CD} 20^{+}\right)$in crypt epithelium and a decrease of B-lymphocytes in surface epithelium, extrafollicular area and follicles, a lower density of memory lymphocytes $\left(\mathrm{CD} 45 \mathrm{R} 0^{+}\right)$in extrafollicular area compared to tRT group. A higher density of B-lymphocytes in crypt epithelium in the RTTH group is due to different patients group, while all patients with RTTH presented with history of recurrent infections and in our study the patients in ITH group had mostly obstructive symptoms with no recurrent infections in a history.
Difference from our study, he didn't analyze memory lymphocytes $\left(\mathrm{CD} 45 \mathrm{R} 0^{+}\right)$in crypts and surface epithelium. B-lymphocytes and memory T-lymphocytes are crucial for induction of secondary immune response and they are more abundant in crypts than in surface epithelium due to repeated bacterial stimulation in the RT group.

During disease, also the dendritic cells decrease in the surface epithelium and increase in the crypt epithelium $(16,17)$. The ratio of dendritic cells in surface to crypt epithelium was $1: 1$ for normal tonsils, but dropped to $1: 3$ for diseased tonsils (16). Alatas found the ratio of dendritic cells in surface to crypt $0.5: 1$ in the RT group and $1: 0.8$ in the RTTH group (9).

In patients with RT we measured higher sera concentrations of immunoglobulines (IgM, IgA, IgG) together with a higher concentration of TNF- $\alpha$ in serum compared to the ITH group. There was $\mathrm{T}_{\mathrm{H}}-1$ immune response in tonsillar tissue in patients with RT based on differences between local cytokine concentration TNF- $\alpha$ and IL-4, the local concentration of TNF- $\alpha$ was 40 times higher than in serum. This can be caused by local overproduction due to monocyte -macrophage activation caused by repeated stimulation by the pathogenic agents. The presence of high levels of these cytokines, nevertheless instead of inducing an increase in immunological efficiency may lead to activation and proliferation of endothelial cells and fibroblasts which result in replacement of immunologically active tissue with fibrotic tissue (7).

The higher levels mainly of $\operatorname{IgG}(18,19)$ together with proinflammatory cytokines such as TNF- $\alpha$ in serum (20) in patients with RT are due to persistent bacterial antigenic stimulation We observed no significant changes in subpopulations of lymphocytes in peripheral blood between both patient groups.

\section{Conclusion}

Our study reported a higher inflammatory response in situ in patients with RT - we found a severe degree of fibrosis with a higher accumulation of lymphocytes in the surface epithelium in RT compared to the ITH group. The results were not statistically significant because of small patient group except the immunostaining of IgG under the crypts in RT ( $p=0.041)$.

We measured $\mathrm{T}_{\mathrm{H}}-1$ immune response in the tonsillar tissue in RT in correlation with other studies (21-24). This observation was based on differences between local cytokine concentration TNF- $\alpha$ and IL-4. The local concentration of TNF- $\alpha$ was 40-times higher than in sera. It would be helpful to measure the local cytokine levels in ITH to understand better the aetiology of tonsillar hypertrophy.

In humoral immunity, we measured a higher sera immunoglobulin concentration (mainly IgG) in RT compared to ITH due to persistent bacterial stimulation.

These mentioned results confirmed the focal infection in tonsils of patients with RT. The tonsillectomy must be therefore the only option for surgical treatment in patients with RT. The young children with ITH can be clinically observed and if they don't have history of recurrent infections, the tonsillotomy should be preferred. 


\section{References}

1. Parker NP, Walner DL. Trends in the indications for pediatric tonsillectomy or adenotonsillectomy. Int J Ped Otolaryngtol 2011; 75 (2): 282-285.

2. Erickson BK, Larson DR, St Sauver JL, Meverden RA, Orvidas LJ. Changes in incidence and indications of tonsillectomy and adenotonsillectomy, 1970-2005. Otolaryngol Head Neck Surgery 2009; 140 (6): 894-901.

3. Bhattacharyya N, Lin HW. Changes and consistencies in the epidemiology of pediatric adenotonsillar surgery, 1996-2006. Otolaryngol Head Neck Surg 2010; 143 (5): 680-684.

4. Eisfeld W, Amler S, Deitmer T. Long-therm results following tonsillotomy in children, Laryngorhinootologie 2010; 89 (8): 466-472.

5. Reichel O, Mayr D, Winterhoff J, de la Chaux R, Hagedorn H, Berghaus A. Tonsillotomy or tonsillectomy? - A prospective study comparing histological and immunological findings in recurrent tonsillitis and tonsillar hyperplasia. Eur Arch Otolaryngol 2007; 264 (3): 277-284.

6. van Kempen MJP, Rijkers GT, van Cauwenberger PB. The immune response in adenoids and tonsils. Int Arch Allergy Immunol 2000; 122 (1): $8-19$.

7. Passali D, Damiani V, Passali GC, Passali FM, Boccazzi A, Bellusi L. Structural and immunological characteristics of chronically inflamed adenotonsillar tissue in childhood. Clin Diagn Lab Immunol.2004; 11 (6): 1154-1157.

8. Brodsky L, Moore L, Stanievich JF, Ogra PL. The immunology of tonsils in children: The effect of bacterial load on the presence of $\mathrm{B}$ and T-cell subsets. Laryngoscope 1988; 98 (1): 93-98.

9. Alatas N, Baba F. Proliferating Active Cells, Lymphocyte Subsets and Dendritic Cells in Recurrent Tonsillitis, Arch Otolaryngol Head Neck Surg 2008; 134 (5): 477-483.

10. Church MK, Norn S, Pao GJK, Holgate ST. Non-IgE dependent bacteria-induced histamine release from human lung and tonsillar mast cells. Clin Allergy 1987; 17 (4): 341-353.

11. Modrzynski M, Mierzwinski J, Zawisza E. The occurrence of food allergy and bacteria allergy in patients with tonsilar hypertrophy. Przegl Lek 2004; 61 (12): 1330-1333.

12. Zhang PC, Pang YT, Loh KS. Comparison of histology between recurrent tonsillitis and tonsillar hypertrophy. Clin Otolaryngol 2003; 28 (3): $235-239$.

13. Olofsson K, Hellstrom S, Hammarstrom ML. The surface epithelium of recurrent infected palatine tonsils is rich in gammadelta T cells. Clin Exp Immunol 1998; 111 (1): 36-47.
14. Rosenmann E, Rabovitz R, Schlesinger M. Lymphocyte subsets in human tonsils: the effect of age and infection. Pediatr Allergy Immunol 1998; 9 (3): 161-167.

15. Go M, Kojima T, Takano K, Murata M, Ichimiya S, Tsubota H, Himi T, Sawada N. Expression and function of tight junctions in the crypt epithelium of human palatine tonsils. J Histochem Cytochem 2004; 52 (12): 1627-1638.

16. Brodsky L, Frankel S, Gorfien J, Rossman J, Noble L. The role of dendritic cells in the development of chronic tonsillar disease in children. Acta Otolaryngol Suppl. 1996; 523: 98-100.

17. Gorfien JL, Noble B, Brodsky L. Comparison of the microanatomical distributions of macrophages and dendritic cells in normal and diseased tonsils. Ann Otol Rhinol Laryngol 2001; 110 (2): 173-182.

18. Friday GA Jr, Paradise JL, Rabin BS, Colborn DK, Taylor HF. Serum immunoglobulin changes in relation to tonsil and adenoid surgery. Ann Allergy 1992; 69 (3): 225-230.

19. Moreno PM, Saches M, Sainz M, Gutierez F. Changes in immunological response in tonsillectomized children. Clin Otolaryngol 1992; 17 (5): 380-382.

20. Unal M, Oztürk C, Gorür K. Effect of Tonsillectomy on Serum Concentrations of Interleukins and TNF- $\alpha$ in Patients with Chronic Tonsillitis. ORL 2002; 64 (4): 254-256.

21. Agren K, Andersson U, Litton M, Funa K, Nord-Lander B, Andersson $\mathbf{J}$. The production of immunoregulatory cytokines is localized to the extrafollicular area of human tonsills. Acta Otolaryngol (Stockh) 1996; 116 (3): 477-485.

22. Agren K, Brauner A, Anderson J. Haemophilus influenzae and Streptococcus pyogenes group A challange induce a Th1 type of cytokine response in cells obtained from tonsillar hypertrophy and recurrent tonsillitis. ORL J Otorhinolaryngol Relat Spec1998; 60 (1): 35-41.

23. Komorowska A, Komorowski J, Banasik M, Lewkowicz P, Tchorzewski H. Cytokines locally produced by lymphocytes removed from the hypertrophic nasopharyngeal and palatine tonsils. Int J of Pediatr Otolaryngol 2005; 69 (7): 937-941.

24. Kerakawauchi H, Kurano Y, Mogi G. Immune responses against Streptococcus pyogenes in human palatine tonsils. Laryngoscope 1997; 107 (5): 634-639. 